\title{
¿Qué caminos existen hacia la calidad de servicio en clubes deportivos de baloncesto? Análisis de orientación emprendedora y nivel de competición
}

\author{
What are the paths to quality of service in basketball sports clubs? \\ Analysis of entrepreneurial orientation and level of competition
}

\author{
J.M. Núnez-Pomar y P. Escamilla-Fajardo*
}

Departamento de Educación Física y Deportiva. Universitat de València (España).

\begin{abstract}
Resumen: La orientación emprendedora (compuesta por innovación, asunción de riesgos y proactividad), es entendida como la disposición para afrontar de forma ventajosa los desafíos del entorno y sus rivales, teniendo creciente interés en la investigación y relacionada generalmente con un mayor rendimiento en distintos ámbitos de la organización. El presente estudio, realizado con clubes de baloncesto españoles, analiza la relación existente entre la Orientación Emprendedora, el nivel de competición de los clubes y la calidad de servicio que prestan a sus deportistas y socios/abonados. En este trabajo, en el que se ha utilizado como metodología de análisis fsQCA se han analizado 103 clubes deportivos de baloncesto $(61 \%$ de categoría nacional y 39\% autonómica). Los resultados muestran diferentes caminos para lograr altos niveles de Calidad de Servicio, destacando Asunción de Riesgos y nivel de competición nacional como variables que mayor presencia tienen en las soluciones de altos niveles de Calidad de servicio.

Palabras clave: Orientación emprendedora, clubes deportivos, rendimiento, calidad de servicio, nivel de competición.
\end{abstract}

Abstract: The entrepreneurial orientation (composed of innovation, risk taking and proactivity), is understood as the willingness to face the challenges of the environment and its rivals in an advantageous way, having a growing interest in research and generally related to greater performance in different areas of the organization. This study, carried out with Spanish basketball clubs, analyses the relationship between the Entrepreneurial Orientation, the level of competition of the clubs and the quality of service they provide to their athletes and members/subscribers. In this work, in which the fsQCA analysis methodology has been used, 103 basketball sports clubs have been analysed (61\% national and 39\% regional). The results show different ways to achieve high levels of Service Quality, with Risk Assumption and the level of national competition standing out as the variables that have the greatest presence in solutions with high levels of Service Quality.

Key words: Entrepreneurial orientation, sports clubs, performance, quality of service, level of competition.

\section{Introducción}

En la actualidad nos enfrentamos a un entorno cambiante, con transformaciones rápidas y ciclos de vida muy cortos (Rauch, Wiklund, Lumpkin, y Frese, 2009). En este contexto económico, político y social, una forma significativa por la que las organizaciones deportivas siguen siendo competitivas en su oferta de servicios es por tener altos niveles de orientación emprendedora (Ratten, 2011). Según Covin y Wales (2018), la Orientación Emprendedora es un tema de investigación vibrante al atraer el interés de cada vez más investigadores en el ámbito académico y gestores y directores en el ámbito profesional.

La Orientación Emprendedora (EO) es entendida como el proceso de creación de estrategias que provee a la organización de una base para acciones y decisiones emprendedoras (Rauch et al., 2009). A pesar de ser un área de investigación reciente (Chen, Ho, Wang, y Wu, 2011), el interés por el constructo EO ha sido creciente y significativo. Gran parte de esta importancia viene derivada de su notable relación con

Dirección para correspondencia [Correspondence address]: Paloma Escamilla-Fajardo. Departamento de Educación Física y Deportiva Universitat de València (Espańa). E-mail: paloma.escamilla@uv.es el rendimiento de la organización (Engelen, Gupta, Strenger, y Brettel, 2015; Lee y Sukoco, 2007).

Según Miller y Friesen (1983) está compuesto por tres dimensiones clave: innovación, proactividad y asunción de riesgos.

La innovación es la tendencia de la organización a desarrollar nuevas ideas, a experimentar y la disposición de procesos creativos que puedan originar nuevos productos, servicios, etc. (Fernández-Mesa, Alegre-Vidal, y Chiva-Gómez, 2012).

La proactividad es la cualidad que permite a la organización anticiparse a los cambios del entorno y adquirir, en su caso, una ventaja competitiva. La proactividad es también la dimensión fundamental de la orientación emprendedora para autores como Becherer y Maurer (1999).

Por último, la asunción de riesgos implica el compromiso de grandes recursos de la organización con la expectativa de obtener resultados beneficiosos posteriores, comprometiendo incluso la estabilidad de la organización (Fernández-Mesa et al., 2012)

Estos atributos, no obstante, no cierran el debate sobre las dimensiones que constituyen el constructo. Sin embargo, la perspectiva de un constructo compuesto por tres dimensio- 
nes es ampliamente aceptada (De Clercq, Sapienza, y Crijns, 2005; Richard, Barnett, Dwyer, y Chadwick, 2004; Yoo, 2001), aunque también hay autores que incorporan dimensiones adicionales. Lumpkin y Dess (1996), incorporan la agresividad competitiva (el compromiso con la superación de los rivales) y la autonomía como componentes del constructo. La autonomía ha sido posteriormente considerada también por (Monsen, 2005) como una dimensión constitutiva, entendida como la capacidad de la plantilla para actuar de forma independiente, centrándose en la mejora y obtención de resultados.

A pesar de la discusión previa sobre dimensiones constitutivas, otro aspecto controvertido lo constituye la consideración unidimensional o multidimensional del constructo (Rauch et al., 2009; Wiklund y Shepherd, 2005). Una aproximación unidimensional implicaría asumir que, con independencia de los elementos constitutivos, todos contribuyen igual a la dimensión de resultado, mientras que una aproximación multidimensional nos lleva a considerar la posibilidad de que las distintas dimensiones pueden contribuir de forma distinta al resultado, o incluso que distintas combinaciones de estas dimensiones pueden arrojar resultados iguales (Lisboa, Skarmeas y Saridakis, 2016).

En el ámbito empresarial, la estrecha relación acreditada entre EO y rendimiento de la organización ha motivado un creciente interés académico por el constructo. Esto se ha podido observar en distintos sectores de servicios o de producción como administración y políticas públicas (Karyotakis y Moustakis, 2016), comercio internacional (Thanos, Dimitratos, y Sapouna, 2017) y educación (Diánez-González y Camelo-Ordaz, 2017), pero no ha sido estudiado de forma tan incisiva en el sector deportivo, donde son escasos los trabajos que abordan esta relación (Núñez-Pomar, Prado-Gascó, Añó, Crespo, y Calabuig, 2016), y menos aún si se trata del sector asociativo deportivo (Ratten, 2011).

El tejido asociativo es de destacada importancia e imprescindible para comprender el fenómeno deportivo en toda su amplitud. Los elementos constitutivos esenciales de dicho tejido son las asociaciones o clubes deportivos, que según el MECD (2017, p.84), son las asociaciones privadas integradas por personas físicas o jurídicas que tengan por objeto la promoción de una o varias modalidades deportivas, la práctica de las mismas por sus asociados, asi como la participación en actividades y competiciones deportivas.

Entre las características destacadas de los clubes deportivos se podría subrayar la diversidad social que atiende, así como la estrecha relación que mantiene con las instituciones (Puig, Moreno, y López, 2010), ya que actualmente son los proveedores de primer orden en la oferta de servicios de ocio, deporte y programas sociales (Wicker y Breuer, 2015). La máxima categoría en la que milita el club deportivo también es un rasgo distintivo, al poder diferenciar un club entre internacional, na- cional, autonómico y local. Dejando los extremos de internacional y local como las categorías en las que existe una menor representación, el grueso del tejido asociativo se encuentra englobado en las categorías nacional y autonómica.

Por otro lado, y entre las diferentes disciplinas deportivas importantes en cuanto al número de clubes deportivos y licencias federativas, el baloncesto español es un ejemplo típico de un caso de éxito. Ocupa el segundo lugar en el ranking FIBA en categoría masculina y femenina, solo superado en ambos casos por Estados Unidos, siendo, por tanto, los líderes a nivel europeo en ambos casos. En el caso del ranking masculino, se trata de una clasificación de un total de 147 países, mientras que, en el femenino, el segundo puesto corresponde a un ranking que recoge a un total de 79 selecciones nacionales (FIBA, 2018). Esta es una de las principales razones por las que se ha escogido esta disciplina para la realización del estudio.

El objetivo del presente estudio es conocer los distintos caminos existentes para predecir la calidad de servicio en clubes deportivos nacionales de baloncesto teniendo en cuenta la Orientación Emprendedora y el nivel de competición mediante la metodología novedosa fsQCA.

\section{Método}

\section{Participantes}

La muestra del presente estudio consiste en 103 clubes deportivos españoles de baloncesto, de los cuales un $61 \%$ serían de categoría nacional y 39\% de categoría autonómica.

\section{Instrumento}

Para la realización de este estudio se utilizó un instrumento compuesto por la Escala de Orientación Emprendedora ( $\alpha$ $=0.80)$, creada por Covin y Slevin (1989) y adaptada por Engelen et al. (2015) y Lee y Sukoco (2007). Dicha escala está compuesta por 8 ítems distribuidos en tres dimensiones: asunción de riesgos $(\alpha=0.71)$, innovación $(\alpha=0.67)$ y proactividad ( $\alpha$ $=0.62)$, y por la Escala de Calidad de Servicio $(\alpha=0.78)$, adaptación realizada de la creada por Vorhies y Morgan (2005), compuesta por 5 ítems y que evalúa el rendimiento de la organización (calidad de servicio). Ambas escalas poseen adecuadas propiedades psicométrica en estudios anteriores (Engelen et al., 2015), lo cual se ha observado también en el presente estudio. Por último, la variable nivel máximo de categoría tiene como respuesta nivel autonómico o nacional.

\section{Procedimiento}

Este estudio se realiza en el contexto de un convenio de colaboración firmado por la Universidad de Valencia (UV) y la 
Federación Española de Baloncesto (FEB). En él se facilita la cooperación de la propia FEB en el envío del cuestionario online a la relación de clubes a nivel nacional. De igual modo, cada Federación Autónoma de Baloncesto envía el formulario a los clubes de su territorio. El cuestionario se contesta a través de la aplicación LimeSurvey (C), la cual genera un link para su cumplimentación. Este únicamente puede ser contestado por una persona de la dirección o cuerpo técnico del club deportivo.

\section{Análisis estadístico}

Para el análisis, la metodología utilizada en este estudio fue el fsQCA, creado por Eng y Woodside (2012) . Este análisis cualitativo comparativo es una forma de análisis basada en la teoría de conjuntos que hace posible el análisis profundo de cómo determinadas condiciones causales (variables explicativas) contribuyen a un determinado resultado (fenómeno a explicar). Esta metodología presupone que cuando se conjugan varios atributos para producir un resultado, la forma en que se combinan esos atributos es más importante que los propios niveles de cada uno de los atributos.

En primer lugar, se realizó una estimación de los análisis descriptivos de los clubes deportivos, para seguidamente, calcular los valores de calibración para fsQCA y realizar el análisis cualitativo de conjuntos difusos (fsQCA). Para ello, los datos que se obtuvieron de las respuestas se transformaron en respuestas adaptadas para esta metodología. Como está sugerido en la literatura previa, se eliminaron todos los valores perdidos y se recalibraron las variables. Nivel de competición (autonómico=0; nacional=1). Las dimensiones de Asunción de riesgos (RIES), Innovación (INNO), Proactividad (PROAC) y Calidad de servicio (CS), fueron recalibradas por el programa fsQCA 2.5 de Claude y Christopher (2014), lo que se logró multiplicando lo ítems que lo componían para posteriormente considerar los tres umbrales propuestos por Woodside (2013): el percentil 10 (concordancia baja o totalmente fuera del conjunto), el percentil 50 (nivel intermedio de concordancia, es decir, ni dentro ni fuera del conjunto) y el percentil 90 (concordancia baja o totalmente dentro del conjunto).

Una vez recalibrados los datos conforme sugiere la literatura, se utilizaron las pruebas de condición necesarias y suficientes. Según Eng y Woodside (2012), para identificar las condiciones suficientes, el análisis fsQCA involucra dos etapas: primero, un algoritmo de tabla de verdad que convierte las puntuaciones de pertenencia de los grupos difusos en una tabla de verdad que enumera todas las posibles combinaciones lógicas de condiciones causales y el resultado empírico de cada configuración. En segundo lugar, el análisis fsQCA genera tres posibles soluciones: complejas, parsimoniosas e intermedias. Ragin (2008) recomienda la última, la cual se presenta en este estudio.

Basándonos en Eng y Woodside (2012), en la metodología
fsQCA un modelo es informativo cuando la consistencia está por encima de .75 . En este sentido el modelo resultante parece adecuado al tener una consistencia de .81 en altos niveles de CS y .77 en bajos niveles de CS.

\section{Resultados}

El primer paso fue calcular los estadísticos descriptivos y los valores de calibración de las variables para su transformación en condiciones difusas (Tabla 1).

Tabla 1. Descripciones principales y calibrados.

\begin{tabular}{|c|c|c|c|c|c|}
\hline & & RIES & INNO & PROAC & CS \\
\hline Media & & 152 & 92.04 & 17.5922 & 5294.66 \\
\hline DT & & 88.49 & 77.25 & 11.90439 & 3710.21 \\
\hline Mín. & & 6 & 4 & 1 & 108 \\
\hline Máx. & & 343 & 343 & 49 & 16807 \\
\hline \multicolumn{6}{|c|}{ Valores calibrados } \\
\hline \multirow[t]{3}{*}{ Percentiles } & 10 & 36 & 20 & 4 & 1155 \\
\hline & 50 & 144 & 64 & 16 & 4500 \\
\hline & 90 & 294 & 216 & 35.60 & 10584 \\
\hline
\end{tabular}

RIES-Asunción de riesgo; INNO-Innovación; PROA—Proactividad; CS—Calidad de Servicio

Es preciso conocer si alguna condición causal es necesaria para el alto grado de CS y para bajos niveles de CS (denominada por la presencia y ausencia de " $\sim$ ", respectivamente) de los resultados. Basándonos en los resultados obtenidos ninguna de las variables analizadas parece ser una condición necesaria tanto para que tenga lugar Calidad de servicio o para que no, ya que la consistencia de las condiciones son inferiores a 0.90 (Ragin, 2008). Sin embargo, para altos valores de CS, la condición más importante sin llegar a ser necesaria es altos niveles de innovación (Cons=.73; $\mathrm{Cob}=.73$ ), mientras que la condición más importante para bajos niveles de SQ, aunque sin ser necesaria sería baja innovación (Cons=.75; Cob=.74) (Tabla 2).

Tabla 2. Condiciones necesarias de SQ.

\begin{tabular}{lllll}
\hline & \multicolumn{2}{l}{ Calidad de Servicio } & \multicolumn{2}{c}{ Calidad de Servicio } \\
\hline & Cons & Cob & Cons & Cob \\
\hline RIES & 0,35 & 0,44 & 0,43 & 0,56 \\
-RIES & 0,65 & 0,52 & 0,57 & 0,48 \\
INN & 0,73 & 0,73 & 0,49 & 0,52 \\
-INN & 0,52 & 0,50 & 0,75 & 0,74 \\
PROA & 0,65 & 0,68 & 0,49 & 0,54 \\
-PROA & 0,56 & 0,52 & 0,71 & 0,68 \\
AUT & 0,65 & 0,68 & 0,51 & 0,55 \\
NAC & 0,57 & 0,53 & 0,70 & 0,68 \\
\hline
\end{tabular}

Nota: Cons: consistencia; Cob: cobertura; $-:$ ausencia de condición; Condición necesaria: consistencia $\geq .90$ 
Por último, teniendo en cuenta las condiciones suficientes, podemos observar como en la tabla de verdad el límite es 1 y el límite de consistencia 0.753 . La representación gráfica utilizada es la utilizada por Fiss (2011) para presentar los re- sultados, en la que los círculos negros indican la presencia de una condición, y los círculos blancos indican la ausencia de una condición (Tabla 3).

Tabla 3. Tres condiciones principales suficientes (solución intermedia).

\begin{tabular}{|c|c|c|c|c|c|c|}
\hline \multirow{3}{*}{$\begin{array}{l}\text { Interrupción de frecuencia: } 1 \text {; todas las condiciones están presentes para la presencia de } \\
\text { las condiciones de resultado y ausentes para la ausencia de la condición de resultado. (-) }\end{array}$} & \multicolumn{3}{|c|}{ Calidad de Servicio } & \multicolumn{3}{|c|}{ - Calidad de Servicio } \\
\hline & \multicolumn{3}{|c|}{ Corte de consistencia: .76} & \multicolumn{3}{|c|}{ Corte de consistencia: .83} \\
\hline & 1 & 2 & 3 & 1 & 2 & 3 \\
\hline RIES & $\bullet$ & $\bullet$ & $\bullet$ & ○ & $\circ$ & $\circ$ \\
\hline INNO & $\bullet$ & & $\bullet$ & & & $\bullet$ \\
\hline PROAC & & $\bullet$ & $\circ$ & & $\bullet$ & \\
\hline $\mathrm{NC}$ & $\bullet$ & $\bullet$ & & $\circ$ & & \\
\hline Consistencia & .81 & .85 & .78 & .74 & .81 & .83 \\
\hline Cobertura bruta & .40 & .38 & .31 & .44 & .41 & .37 \\
\hline Cobertura única & .01 & .01 & .04 & .14 & .04 & .04 \\
\hline Consistencia general de la solución & & & .81 & & & .77 \\
\hline Cobertura general de la solución & & & .57 & & & .61 \\
\hline
\end{tabular}

Los resultados obtenidos muestran como distintas combinaciones de condiciones pueden derivar en altos niveles de Calidad de Servicio, una de las dimensiones de rendimiento en clubes deportivos (Tabla 1). Los resultados exponen un modelo compuesto por cuatro soluciones que explican el 57\% de los casos con altos niveles de Calidad de servicio (solution coverage: .572391; solution consistency: .807037). El camino más importante estaría compuesto por altos niveles de RIES e INNO en clubes deportivos nacionales (raw coverage $=.40$; unique coverage $=.01$; consistency $=.81$ ); altos niveles de RIES y PROAC en clubes deportivos nacionales (raw coverage $=.38$; unique coverage $=.01$; consistency $=.85$ ) y el tercer camino en orden de importancia sería altos niveles de INNO y RIES y bajos niveles de PROAC (raw coverage $=.31$; unique coverage $=$ .04 ; consistency= .78), explicando el $40 \%, 38 \%$ y $31 \%$ de los casos de altos niveles de calidad de servicio respectivamente.

Sin embargo, existen tres caminos que explican un $61 \%$ del total de casos de bajos niveles de Calidad de Servicio (solution coverage: .613026; solution consistency: .769727). El camino más explicativo dentro del modelo sería bajos niveles de RIES en clubes deportivos autonómicos (raw coverage $=.44$; unique coverage $=.14$; consistency $=.74$ ), seguido por bajos niveles de RIES y altos niveles de PROAC (raw coverage $=.41$; unique coverage $=.04$; consistency $=.81$ ), mientras que el último camino explicativo serían bajos niveles de RIES $\mathrm{y}$ altos niveles de INNO (raw coverage $=.37$; unique coverage $=$ .04 ; consistency $=.83$ ), explicando un $44 \%, 41 \%$ y $37 \%$ del total de casos con bajos niveles de Calidad de servicio respectivamente.

\section{Discusión y conclusiones}

Este estudio explora los diferentes caminos existentes para altos y bajos niveles de la variable de rendimiento de la organización, Calidad de servicio mediante el modelo fsQCA. El análisis de la Orientación Emprendedora ha crecido en importancia debido a la relación positiva y directa que tiene con el rendimiento de la organización, tanto de forma general (Covin, Green, y Slevin, 2006), como alguna de sus dimensiones como la innovación (Hult, Hurley, y Knight, 2004).

Las organizaciones que muestran altos niveles de $\mathrm{EO}$ son capaces de responder rápidamente a los cambios del entorno, mejorando su posición competitiva y resultado frente a sus competidores (Meliá y Pérez, 2005). En el presente estudio la Orientación Emprendedora ha sido tratada como variable multidimensional, englobando las dimensiones de Asunción de riesgos, Innovación y Proactividad. La dimensionalidad del constructo ha sido debatida de forma incipiente por diferentes autores, aunque basándonos en diferentes meta-análisis la EO ha sido estudiada principalmente de forma multidimensional (Rauch et al., 2009; Wiklund y Shepherd, 2005).

Sobre la base de los resultados obtenidos, ninguna de las condiciones es necesaria para altos niveles de CS, al ser inferiores a 0.90 (Ragin, 2008). Sin embargo, la innovación es la condición más importante sin llegar a ser necesaria, al igual que bajos niveles de innovación es la condición más importante para bajos niveles de Calidad de servicio. En este sentido, algunos autores consideran que la innovación es la única que define la orientación emprendedora de la organización 
(Hult, Snow, y Kandemir, 2003) o incluso que lo que distingue a una organización empresarial de una organización no empresarial es el uso de la innovación (Schuler, 1986).

$\mathrm{Si}$ analizamos los resultados obtenidos, existen 3 soluciones que explican un $57 \%$ de Calidad de Servicio (RIES*INNO*NAC; RIES*PROAC*NC; RIES*INNO -PROAC). De forma global y basándonos en los caminos anteriores, cabe destacar que la mayor presencia la encontramos en Asunción de riesgos ya que es condición suficiente en las tres soluciones para altos niveles de Calidad de servicio, y deben existir bajos niveles de RIES en los tres caminos más importantes para bajos niveles generales de CS. Por ello, teniendo en cuenta estos resultados, como estrategia de gestión, sería más importante focalizar la atención en la Asunción de riesgos de la organización respecto al resto. La Asunción de riesgos se entiende como la aplicación de medidas audaces, la captación de grandes cantidades de financiación y/o la asignación de recursos significativos a la organización, implica la disposición a comprometer recursos significativos para las oportunidades futuras que se pueden generar. Es importante señalar que debe haber una posibilidad razonable de fracaso, ya que de esta forma se compromete la estabilidad de la organización (Fernández-Mesa et al., 2012).

En la misma línea de análisis, la dimensión de Innovación está presente en dos de las soluciones más importantes para altos niveles de Calidad de servicio. La innovación en una organización puede ser entendida como la introducción de un nuevo producto o servicio, una nueva producción o una nueva estructura o sistema administrativo (Hult et al., 2004) .

La Proactividad se encuentra presente en uno de los caminos para altos niveles de CS y ausente en otro de los caminos, mientras que deben existir altos niveles de PROAC en otra de las soluciones para bajos niveles de CS. Por tanto, y basándonos en las estrategias de gestión, la proactividad es la dimensión menos importante de la Orientación emprendedora en la influencia positiva en la Calidad de Servicio. Esto cobra sentido si nos basamos en Lumpkin y Dess (1996, p.153) quienes afirman que "la actividad o los procesos empresariales pueden, a veces, conducir a resultados favorables en una dimensión de rendimiento y a resultados desfavorables en una dimensión de rendimiento diferente".

Otra de las variables tenidas en cuenta en el presente estudio ha sido el nivel de competición, siendo ésta una variable intrínseca de los clubes deportivos. El nivel de competición ha sido tratado en la literatura de la gestión del deporte como una variable a considerar a la hora de estudiar aspectos organizativos de las entidades deportivas (Nagel, Schlesinger, Bayle, y Giauque, 2015), como es el caso del nivel de profesionalización o las crecientes tensiones para que los clubes y federaciones consigan mejorar su rendimiento en un entorno cada vez más exigente. Los mismos autores señalan diversos retos que afrontan en la actualidad federaciones y clubes de- portivos, entre los que destacamos la orientación al servicio, la facilitación y flexibilización de las condiciones de acceso, y la gestión de la calidad, aspectos que parecen estar convirtiéndose en importantes herramientas para posibilitar el rendimiento de las organizaciones deportivas (Nagel et al., 2015, p. 408). Powell y Friedkin (1987) ya señalaban la creciente tensión a la que se sometían las organizaciones sin ánimo de lucro de mejorar su rendición de cuentas y eficiencia, con el fin de fortalecer su legitimidad y posición, recurriendo en ocasiones a personas con experiencia y credenciales de gestión, y mejorando sus capacidades administrativas.

En este contexto, los estudios señalan normalmente el nivel de la organización como aspecto definitorio a tener en cuenta (clubes de nivel internacional, federaciones nacionales o internacionales, etc.), lo que constituye una evidencia de que el nivel de competición en el que opera la organización constituye una variable determinante para caracterizarla. Por ello, si tenemos en cuenta el nivel más alto en el que juega el club deportivo como punto de referencia, nos permite diferenciar entre ámbito nacional y regional. Basándonos en los resultados obtenidos, en dos de las soluciones, ser un club militando en categoría nacional es una condición suficiente para alto niveles de Calidad de Servicio. No debemos olvidar que el Nivel de competición podría ser considerado una variable de rendimiento en sí misma, por lo que puede resultar normal que las organizaciones de categoría nacional presenten resultados más cercanos a la Calidad de servicio.

A modo de conclusión se puede extraer que la Orientación Emprendedora ayuda a las organizaciones a traducir las posibilidades de un entorno generoso en mayores niveles de rendimiento (Engelen et al., 2015; Rosenbusch, Rauch, y Bausch, 2013). Basándonos en esta relación corroborada en la literatura previa, nuestro estudio sugiere que esta correlación también existe en los clubes deportivos, afirmación corroborada en base a los resultados obtenidos.

Existen diferentes caminos hacia niveles altos de calidad de servicio teniendo en cuenta la Orientación Emprendedora de forma multidimensional (innovación, asunción de riesgos y proactividad) y el nivel de competición (nacional o autonómica). La asunción de riesgos es la dimensión más importante en las soluciones que conducen a un mejor rendimiento (CS), mientras que la proactividad es la dimensión menos necesaria para llegar a altos niveles de rendimiento de la organización.

De igual modo, empleando y adaptando a nuestro entorno de investigación la definición de Anderson, Kreiser, Kuratko, Hornsby Eshima (2015), se puede señalar que es importante que los clubes deportivos muestren una alta orientación emprendedora. Esta actitud implica la lucha por introducir constantemente nuevos servicios, estrategias y modelos, implica evitar el estancamiento y buscar constantemente nuevos espacios en el mercado, y mejorar así su posición en un entorno competitivo. 


\section{Limitaciones y futuras líneas de investigación}

\section{Limitaciones del estudio}

El estudio ha contado con limitaciones al contar con una escasa muestra teniendo en cuenta la población objeto de estudio (clubes deportivos nacionales de baloncesto en España). La muestra corresponde a todo el territorio nacional pero no contamos con un porcentaje real de cada comunidad autónoma.

\section{Futuras líneas de investigación}

Como futura línea de investigación estaría aumentar el tamaño de la muestra y hacer una comparativa de metodologías y análisis de resultados. Además, sería interesante analizar variables moderadoras que pueden influir en la relación existente entre Orientación Emprendedora y diferentes variables de rendimiento de la organización como puede ser Calidad de Servicio, Rendimiento Económico, Social y Deportivo.

\section{Referencias}

1. Anderson, B. S., Kreiser, P. M., Kuratko, D. F., Hornsby, J. S., y Eshima, Y. (2015). Reconceptualizing entrepreneurial orientation. Strategic Management Journal, 36(10), 1579-1596.

2. Becherer, R. C., y Maurer, J. G. (1999). The proactive personality disposition and entrepreneurial behavior among small company presidents. Journal of small business management, 37(1), 28-36.

3. Chen, J. K., Ho, Y.-S., Wang, M.-H., y Wu, Y.-R. (2011). Perspective research entrepreneurship output performance in 1992-2009. En Technology Management in the Energy Smart World (PICMET), 10(3), 1-10

4. Claude R y Christopher R (2014) acq [Computer Programme], Version 2.1.12. Houston, TX: University of Houston-Downtown.

5. Covin, J. G., Green, K. M., y Slevin, D. P. (2006). Strategic process effects on the entrepreneurial orientation-sales growth rate relationship. Entrepreneurship theory and practice, 30(1), 57-81.

6. Covin, J. G., y Slevin, D. P. (1989). Strategic management of small firms in hostile and benign environments. Strategic Management Journal, 10(1), 75-87. https://doi.org/10.1002/smj.4250100107

7. Covin, J. G., y Wales, W. J. (2018). Crafting High-Impact Entrepreneurial Orientation Research: Some Suggested Guidelines. SAGE Publications Sage CA: Los Angeles, CA.

8. De Clercq, D., Sapienza, H. J., y Crijns, H. (2005). The internationalization of small and medium-sized firms. Small business economics, 24(4), 409-419.

9. Diánez-González, J. P., y Camelo-Ordaz, C. (2017). The influence of the structure of social networks on academic spin-offs' entrepreneurial orientation. Industrial Marketing Management.

10. Eng, S., y Woodside, A. G. (2012). Configural analysis of the drinking man: Fuzzy-set qualitative comparative analyses. Addictive Behaviors, 37(4), 541-543.

11. Engelen, A., Gupta, V., Strenger, L., y Brettel, M. (2015). Entrepreneurial orientation, firm performance, and the moderating role of transformational leadership behaviors. Journal of Management, 41(4), 1069-1097.

12. Fernández-Mesa, A., Alegre-Vidal, J., y Chiva-Gómez, R. (2012). Orientación emprendedora, capacidad de aprendizaje organizativo y desempeño innovador. Journal of technology management y innovation, $7(2), 157-170$.

13. FIBA (2018). FIBA World Ranking. Switzerland: Fédération Internationale de Basketball. http://www.fiba.basketball/es/rankingmen

14. FIBA (2018). FIBA World Ranking. Switzerland: Fédération Internationale de Basketball. http://www.fiba.basketball/es/rankingwomen

15. Fiss, P. C. (2011). Building better causal theories: A fuzzy set approach to typologies in organization research. Academy of Management Journal, 54(2), 393-420.

16. Hult, G. T. M., Hurley, R. F., y Knight, G. A. (2004). Innovativeness: Its antecedents and impact on business performance. Industrial marketing management, 33(5), 429-438.
17. Hult, G. T. M., Snow, C. C., y Kandemir, D. (2003). The role of entrepreneurship in building cultural competitiveness in different organizational types. Journal of management, 29(3), 401-426.

18. Karyotakis, K. M., y Moustakis, V. S. (2016). Organizational factors, organizational culture, job satisfaction and entrepreneurial orientation in public administration. The European Journal of Applied Economics, 13(1), 47-59.

19. Lee, L. T.-S., y Sukoco, B. M. (2007). The Effects of Entrepreneurial Orientation and Knowledge Management Capability on Organizational Effectiveness in Taiwan: The Moderating Role of Social Capital. International Journal of Management; Poole, 24(3), 549-572.

20. Lisboa, A., Skarmeas, D., y Saridakis, C. (2016). Entrepreneurial orientation pathways to performance: A fuzzy-set analysis. Journal of business research, 69(4), 1319-1324.

21. Lumpkin, G. T., y Dess, G. G. (1996). Clarifying the entrepreneurial orientation construct and linking it to performance. Academy of management Review, 21(1), 135-172.

22. Meliá, M. R., y Pérez, A. B. (2005). Relación entre la orientación al mercado y la orientación emprendedora: su influencia en el rendimiento de la empresa. Revista europea de dirección y economía de la empresa, 14(3), 165-180.

23. Miller, D., y Friesen, P. H. (1983). Strategy-making and environment: the third link. Strategic management journal, 4(3), 221-235.

24. MECD: Ministerio de Educación, Cultura y Deporte. Gobierno de España (2017). Anuario de estadísticas Deportivas 2017. Recuperado de http://www.csd.gob.es/csd/sociedad/anuario-de-estadisticas-deportivas-2017/

25. Monsen, E. (2005). Employees Do Matter: Autonomy, Teamwork and Corporate Entrepreneurial Culture (PhD Thesis). University of Colorado.

26. Nagel, S., Schlesinger, T., Bayle, E., y Giauque, D. (2015). Professionalisation of sport federations-a multi-level framework for analysing forms, causes and consequences. European sport management quarterly, 15(4), 407-433

27. Núñez-Pomar, J., Prado-Gascó, V., Añó, V., Crespo, J., y Calabuig, F. (2016). Does size matter? Entrepreneurial orientation and performance in Spanish sports firms. Journal of Business Research, 69(11), 5336-5341.

28. Powell, W. W., y Friedkin, R. (1987). Organizational change in nonprofit organizations. The nonprofit sector: A research handbook, 180-192.

29. Puig, N., Moreno, A., y López, C. (2010). Proposal of an interpretative theoretical frame about sport associationism in Spain. European Journal of Human Movement, 2, 75-92.

30. Ragin, C. C. (2008). Redesigning social inquiry: Fuzzy sets and beyond (Vol. 240). Wiley Online Library.

31. Ratten, V. (2011). Sport-based entrepreneurship: towards a new theory of entrepreneurship and sport management. International entrepreneurship and management journal, 7(1), 57-69.

32. Rauch, A., Wiklund, J., Lumpkin, G. T., y Frese, M. (2009). Entrepre- 
neurial orientation and business performance: An assessment of past research and suggestions for the future. Entrepreneurship theory and practice, 33(3), 761-787.

33. Richard, O. C., Barnett, T., Dwyer, S., y Chadwick, K. (2004). Cultural diversity in management, firm performance, and the moderating role of entrepreneurial orientation dimensions. Academy of management journal, 47(2), 255-266.

34. Rosenbusch, N., Rauch, A., y Bausch, A. (2013). The mediating role of entrepreneurial orientation in the task environment-performance relationship: A meta-analysis. Journal of Management, 39(3), 633-659.

35. Schuler, R. S. (1986). Fostering and facilitating entrepreneurship in organizations: Implications for organization structure and human resource management practices. Human resource management, 25(4), 607-629.

36. Thanos, I. C., Dimitratos, P., y Sapouna, P. (2017). The implications of international entrepreneurial orientation, politicization, and hostility upon SME international performance. International Small Business Journal, 35(4), 495-514.
37. Vorhies, D. W., y Morgan, N. A. (2005). Benchmarking marketing capabilities for sustainable competitive advantage. Journal of marketing, 69(1), 80-94.

38. Wicker, P., y Breuer, C. (2015). How the economic and financial situation of the community affects sport clubs' resources: Evidence from multi-level models. International Journal of Financial Studies, 3(1), $31-48$.

39. Wiklund, J., y Shepherd, D. (2005). Entrepreneurial orientation and small business performance: a configurational approach. Journal of business venturing, 20(1), 71-91.

40. Woodside, A. G. (2013). Moving beyond multiple regression analysis to algorithms: Calling for adoption of a paradigm shift from symmetric to asymmetric thinking in data analysis and crafting theory. Journal of Business Research. 66(4), 463-472.

41. Yoo, S.-J. (2001). Entrepreneurial orientation, environmental scanning intensity, and firm performance in technology-based SMEs. Frontiers of Entrepreneurship Research, 365-367. 